\title{
Assessment of Cognitive Brain Function in Ecstasy Users and Contributions of Other Drugs of Abuse: Results from an fMRI Study
}

\author{
Gerry Jager*,', Maartje ML de Win², Ingeborg van der Tweel ${ }^{3}$, Thelma Schilt ${ }^{4}$, Rene S Kahn', \\ Wim van den Brink ${ }^{4}$, Jan $M$ van Ree ${ }^{5}$ and Nick F Ramsey' \\ 'Department of Neurosurgery, Rudolf Magnus Institute of Neuroscience, University Medical Center, Utrecht, The Netherlands; ${ }^{2}$ Department of \\ Radiology, Academic Medical Center, University of Amsterdam, Amsterdam, The Netherlands; ${ }^{3}$ Department of Biology, Center for Biostatistics, \\ Utrecht University, Utrecht, The Netherlands; ${ }^{4}$ Amsterdam Institute for Addiction Research and Department of Psychiatry, Academic Medical \\ Center, University of Amsterdam, Amsterdam, The Netherlands; ${ }^{5}$ Department of Pharmacology and Anatomy, Rudolf Magnus Institute of \\ Neuroscience, University Medical Center, Utrecht, The Netherlands
}

\begin{abstract}
Heavy ecstasy use has been associated with neurocognitive deficits in various behavioral and brain imaging studies. However, this association is not conclusive owing to the unavoidable confounding factor of polysubstance use. The present study, as part of the Netherlands XTC Toxicity study, investigated specific effects of ecstasy on working memory, attention, and associative memory, using functional magnetic resonance imaging $(\mathrm{fMRI})$. A large sample $(n=7 \mathrm{I})$ was carefully composed based on variation in the amount and type of drugs that were used. The sample included 33 heavy ecstasy users (mean 322 pills lifetime). Neurocognitive brain function in three domains: working memory, attention, and associative memory, was assessed with performance measures and $\mathrm{fMRI}$. Independent effects of the use of ecstasy, amphetamine, cocaine, cannabis, alcohol, tobacco, and of gender and IQ were assessed and separated by means of multiple regression analyses. Use of ecstasy had no effect on working memory and attention, but drug use was associated with reduced associative memory performance. Multiple regression analysis showed that associative memory performance was affected by amphetamine much more than by ecstasy. Both drugs affected associative memory-related brain activity, but the effects were consistently in opposite directions, suggesting that different mechanisms are at play. This could be related to the different neurotransmitter systems these drugs predominantly act upon, that is, serotonin (ecstasy) vs dopamine (amphetamine) systems.

Neuropsychopharmacology (2008) 33, 247-258; doi:I0.1038/sj.npp. I30I4I5; published online 25 April 2007
\end{abstract}

Keywords: ecstasy; polysubstance use; fMRI; neuroimaging; cognition; brain function

\section{INTRODUCTION}

Ecstasy (3,4-methylenedioxymethamphetamine, MDMA, XTC) is a popular recreational drug, despite the fact that there is considerable concern about its neurotoxic potential. Studies in animals have shown deleterious effects of MDMA on the serotonin system, indicated by serotonergic axonal injury, decreases in the number of central serotonin transporters (SERT), and a significant depletion of serotonin in various cortical and subcortical regions (for review see Ricaurte et al, 2000; Green et al, 2003). On the basis of these animal data, it is likely that ecstasy might damage serotonin neurons in

*Correspondence: Dr G Jager, Department of Neurosurgery, Rudolf Magnus Institute of Neuroscience, University Medical Center, Heidelberglaan 100, A.01.126, 3584 CX Utrecht, The Netherlands, Tel: + 3130 2509998, Fax: + 31302505443 ,

E-mail: gjager@umcutrecht.nl

Received 24 October 2006; revised 9 February 2007; accepted 6 March 2007 the human brain too. In humans, the literature on the potential neurotoxicity of ecstasy is extensive and complex and it is beyond the scope of this paper to discuss in detail the methods available for assessing the neurotoxic effects of ecstasy on the living human brain or differences in results across studies in different populations (for review see Parrott, 2001; Reneman, 2003; Morton, 2005). In general, however, neuroimaging studies (PET, SPECT) provide evidence for sustained serotonergic dysfunction in people who heavily use ecstasy, whereas the dopaminergic system appears to be unaffected (McCann et al, 1998, 2005; Kish, 2002; Buchert et al, 2004; Gouzoulis-Mayfrank and Daumann, 2006a, b; Parrott, 2006; Reneman et al, 2001, 2006). Whether the alterations in serotonergic function are permanent is not yet clear. Some studies suggest that for instance reductions in SERT, a marker of serotonergic neurotoxicity, are transient in most but not all brain regions, as reversibility was shown in former ecstasy users (Semple et al, 1999; Reneman et al, 2001; Buchert et al, 2004). 
Cognitive consequences of ecstasy use have been examined more extensively. Numerous cross-sectional studies reported impairments of learning and memory in moderate to heavy recreational users (for review see Morgan, 2000; Parrott, 2000; Verbaten, 2003). However, little is known about the effects of ecstasy on the neural systems involved in cognition. The few available functional MRI studies have primarily focused on working memory and generated inconclusive results concerning the effects of ecstasy on brain activity patterns and the specific brain areas that are affected (Daumann et al, 2003, 2004a, b, c, d; Jacobsen et al, 2004; Moeller et al, 2004). One study investigated episodic memory function and suggested that memory deficits in ecstasy users arise from a hippocampal dysfunction (Daumann et al, 2005). Summarizing, there is some evidence of effects of ecstasy use on neurocognitive brain function, but this issue is clearly in need of further investigation.

Research in this area suffers from methodological problems for which there are no easy solutions (Turner and Parrott, 2000). Most human studies contend with interference of many potential confounders, such as polysubstance use and large heterogeneity in recreational ecstasy users (Gouzoulis-Mayfrank and Daumann, 2006a). Experienced ecstasy users not only consume more ecstasy than novice users but they are also more likely to consume other drugs such as cannabis, amphetamine, cocaine, LSD, and psilocybin (mushrooms) (Scholey et al, 2004). Consequently, cumulative ecstasy use almost invariably correlates highly with use of other drugs, making it almost impossible to differentiate between effects of ecstasy and of the other drugs. Indeed, several studies have indicated that signs of neurotoxicity in ecstasy users may well be associated with polydrug use in general or with the use of other drugs, such as cannabis and amphetamine (Daumann et al, 2004a). In some studies, an effort was made to control for the use of these other drugs, either by including a group of 'pure' ecstasy users (Halpern et al, 2004), a group of ecstasy-naive polysubstance users (Rodgers, 2000), or by statistically adjusting for polydrug use (Medina et al, 2005). Although statistical techniques help to separate the effects of ecstasy and of other drugs to some extent, they generally do not suffice if subjects are recruited at random, given the fact that ecstasy use almost invariably is associated with polydrug use.

The present study aims to clarify the specific effects of ecstasy on neurocognitive brain function, that is, effects that can be ascribed to ecstasy and not to the co-occurring use of other drugs. For this purpose, a large sample $(n=71)$ with substantial variation in the amount and type of drug used was carefully composed. Multiple regression analysis with ecstasy and other drugs as separate regressors was applied to investigate the specific effects of ecstasy on working memory, selective attention, and associative memory, measured with functional magnetic resonance imaging (fMRI). We hypothesized that if deficits in memory or attention and/or related brain function would surface, ecstasy use would be the primary but not the only factor accounting for these abnormalities. We expected that amphetamines, cannabis, and/or cocaine would contribute to possible neurocognitive deficits.

\section{MATERIALS AND METHODS}

This study is part of the Netherlands XTC Toxicity (NeXT) study. A detailed description of the design and objectives of the NeXT study is provided in a paper on the methods (De Win et al, 2005). Besides fMRI, subjects underwent SPECT and MR imaging and cognitive testing; results of these measurements will be reported in separate publications (De Win et al, 2007; Schilt et al, in press). In two previous papers, we reported fMRI findings in a part of the nonecstasy using subjects $(n=27)$ on the effects of cannabis on cognitive brain function (Jager et al, 2006, 2007).

\section{Subjects}

A total of 71 subjects ( 44 male, 27 female; mean age 23 years (SD 3.8, range 18-37) were included based on specific variations in the amount and type of drugs they were using and with the objective to keep correlations between the drugs that were used as low as possible. Potential candidates interested to participate in the study were asked to fill out a questionnaire on their drug use, but were blind to the inclusion criteria. Besides the typical heavy polysubstance ecstasy users, preference was given to candidates who were either 'selective ecstasy users' (100 ecstasy pills or more lifetime, but no or almost no use of other drugs except for cannabis) or 'polydrug-but-no-ecstasy users' (extensive experience with amphetamine and/or cocaine, but (almost) no ecstasy use, that is $<10$ pills lifetime). Eventually, the sample included 33 heavy ecstasy users and 38 non-ecstasy users, with both ecstasy users and non-ecstasy users showing considerable variation in type and amount of other drugs taken, for example, some heavy ecstasy users reported minimal use of other drugs such as cannabis, amphetamine, or cocaine, whereas others were moderate or frequent users of one or more other psychoactive drug.

All subjects were right-handed and were excluded if they reported: major medical, neurological, or psychiatric disorders; current use of psychotropic medications; use of intravenous drugs; pregnancy; and contra-indications for MRI. Except for smoking, which was allowed until $2 \mathrm{~h}$ before scanning, subjects had to abstain from use of all psychoactive drugs for at least 2 weeks and from alcohol for at least 1 week before examinations. Compliance to abstinence was checked with urine drug screening (enzyme-multiplied immunoassay for amphetamines, ecstasy, opiates, cocaine, benzodiazepines, cannabis, and alcohol). Hair samples were collected for drug hair analysis on previous ecstasy use (gas chromatography/mass spectroscopy).

Subjects were paid for their participation ( $€ 150$ for 2 days) and gave their written consent according to the Helsinki Declaration. The local medical ethics committee approved the study.

\section{Procedure}

All 71 subjects completed validated self-report questionnaires about their drug use (Van den Wijngaart et al, 1997). They were screened for axis I psychiatric disorders using the Dutch version of the Mini International Neuropsychiatric Interview for DSM-IV clinical disorders (Sheehan et al, 1998). Urine samples were collected on the day of 
scanning and verbal intelligence was estimated using the Dutch version of the National Adult Reading Test (Schmand et al, 1991).

\section{Assessment of Working Memory, Selective Attention, and Associative Memory}

Three fMRI tasks were administered: a modified Sternberg item recognition task (denoted STERN), a visuo-auditory selective attention task (SAT), and a pictorial associative memory task that depends on (para)hippocampal brain function (PMT). The STERN task has the following basic format: a set of five consonants is shown for $5340 \mathrm{~ms}$ (the

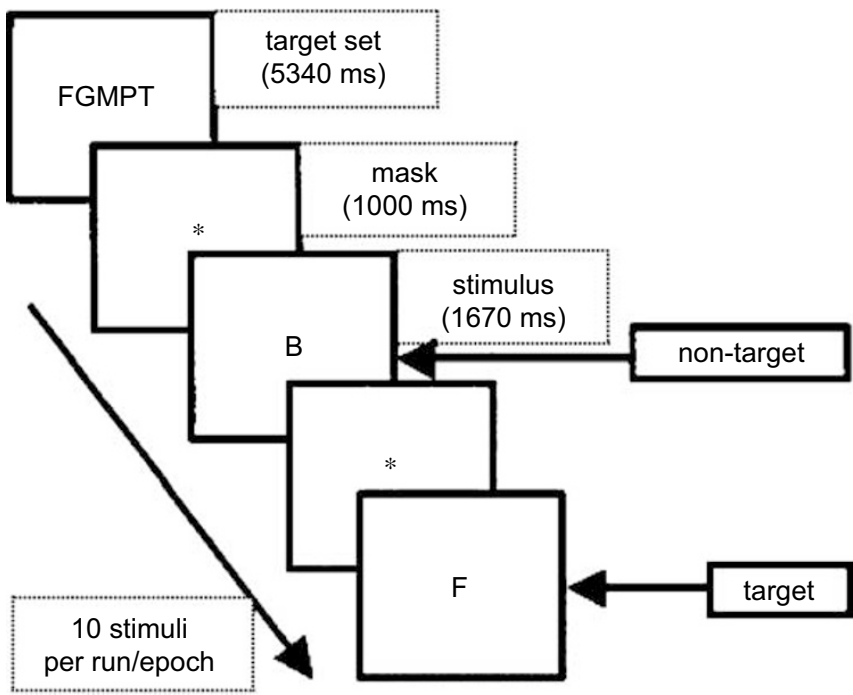

Figure I The temporal sequence of events is shown for the STERN task. Each epoch starts with presentation of the target-set, and is followed by ten trials. Subjects have to press a button as fast as possible, if the letter belongs to the target-set. target-set). After this, a series of 10 consonants is displayed in sequence (Figure 1). Subjects are instructed to memorize the target-set, and subsequently press a button as quickly as possible when a consonant belongs to the target-set $(50 \%$ were targets). Two experimental tasks were administered, which differed only with regard to the target-set(s): a novel set and a practiced set. In the practiced set task (PT), the same set was used repeatedly. In the novel set task (NT), the composition of the target-set was changed after every run of 10 trials. An additional reaction time control task (CT) was included, as well as rest periods of equal epoch duration (for further details on the STERN task see Jansma et al, 2001; Ramsey et al, 2004).

The second task SAT is a visuo-auditory oddball detection task. It involves detection of tones deviant in pitch from a baseline tone, and similarly, detection of dots deviant in size from a baseline dot (Figure 2). A threshold for detecting differences in pitch and dot-size was determined individually before the scan session by adjusting it until the subject detected $80 \%$ of the deviant stimuli. Tones and dots were presented simultaneously in epochs of $29 \mathrm{~s}$ each. Epochs differed only with regard to the task instruction. At the start of each epoch, subjects were instructed to attend either to the tones while ignoring the dots (TO), or vice versa (DO) (for details see Jager et al, 2006).

The PMT task is a pictorial associative memory task, modified from a task paradigm from Henke et al (1997) and involves three task conditions. First, an associative learning phase (AL) is conducted which requires subjects to remember a specific combination of pictures and to establish a meaningful connection between the two pictures. In the next phase, single item pictures have to be classified (SC), which serves as a control task. Finally, in the retrieval phase (RE), subjects have to recognize specific combinations previously presented during associative learning. The RE-task condition enables to measure recall accuracy, which

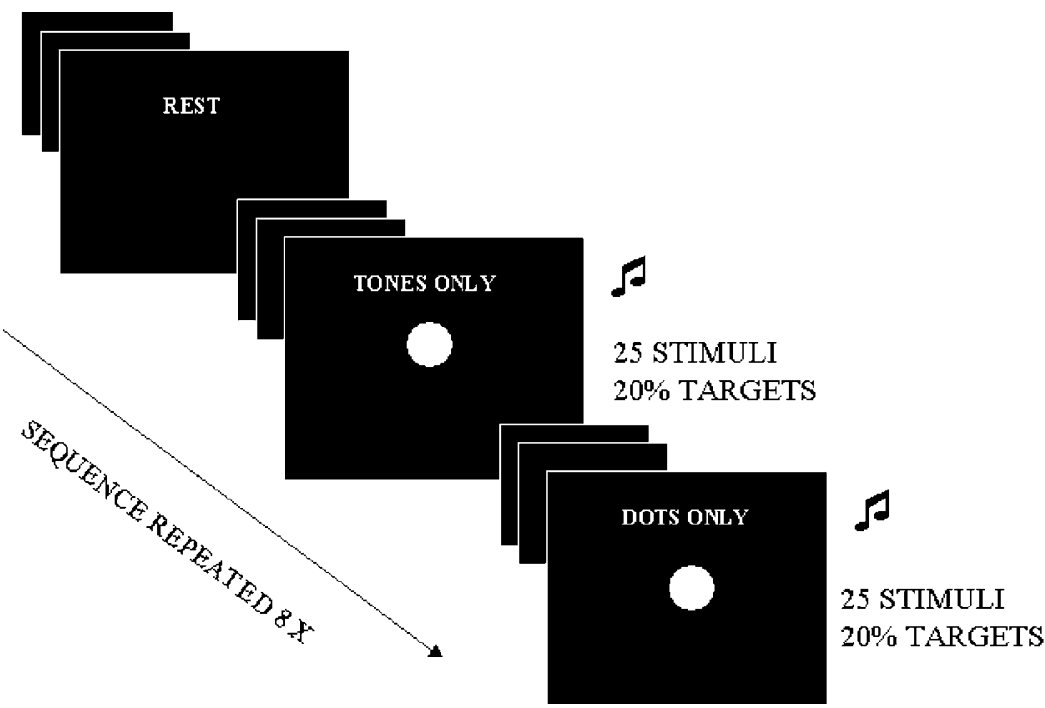

Figure 2 The temporal sequence of events is shown for the SAT task. Each epoch (duration $29 \mathrm{~s}$ ) starts with an instruction slide ( $5 \mathrm{~s}$ ), indicating 'rest', 'attend to the tones only', or 'attend to the dots only'. Both during 'tones only' and 'dots only', the instruction slide is followed by a series of 25 stimuli (simultaneous asynchronous presentation of tones and dots at a variable interstimulus interval rate) of which on average $20 \%$ deviant (targets). In case of a target, subjects have to press a button as fast as possible. Before fMRI scanning, the difference between standard and deviant tones and dots is determined for each individual by changing the contrast until a performance of $80 \%$ correct is obtained. 
serves as an indirect measure for adequate associative learning during the $\mathrm{AL}$ phase. In healthy volunteers, this task reliably reveals brain activity in the hippocampus and parahippocampal gyrus bilaterally, especially during the $\mathrm{AL}$ condition (Henke et al, 1997). Figure 3 depicts a schematic example of the PMT task. In the scanner, each picture, containing two color photographs on a white background, was presented for $5000 \mathrm{~ms}$, followed by a $2340 \mathrm{~ms}$ fixation cross. Each task condition was presented in four epochs of eight stimuli (picture + fixation cross). Also, four rest periods (RS) were included of equal epoch duration. For further details on the PMT task, we refer to Jager et al (2007).

Scans were made on a clinical Philips ACS-NT 1.5 Tesla MR-scanner with PT 6000 gradients, using a standard scan protocol (navigated 3D PRESTO; Ramsey et al, 1998). Voxel size was $4 \mathrm{~mm}$ isotropic. For further details on the scan procedure and scan parameters, we refer to Jansma et al (2001), Ramsey et al (2004) and Jager et al $(2006,2007)$.

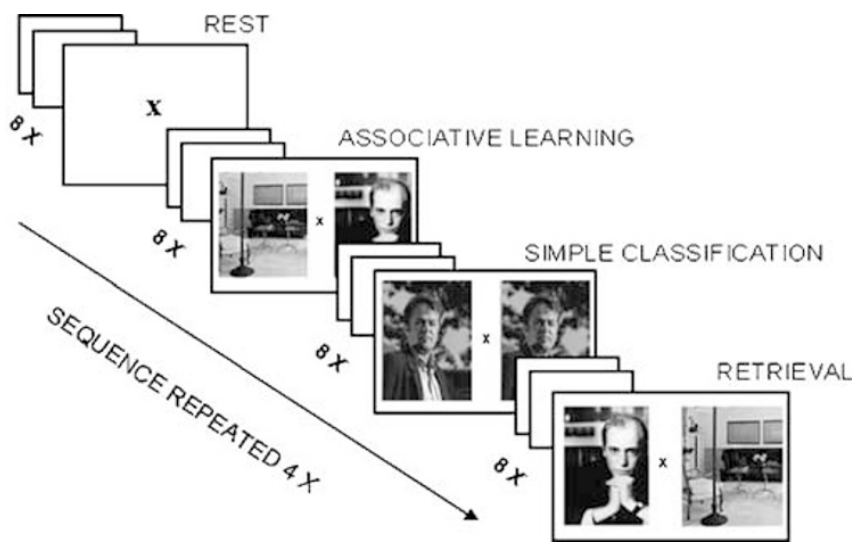

Figure 3 The temporal sequence of events is shown for the PMT task. Each epoch starts with an instruction slide $(5 \mathrm{~s})$ followed by a fixation cross $(2.5 \mathrm{~s})$. This is followed by eight trials of $7.5 \mathrm{~s}$ each (picture pair $5 \mathrm{~s}$, fixation cross $2.5 \mathrm{~s}$ ). Subjects have to respond to the task by pressing one out of two buttons, according to the instruction in each task condition.

\section{Dependent and Independent Variables}

Drug use (independent variables). Table 1 gives a description of the drug use history of the sample. Use of GHB (liquid ecstasy), LSD, psilocybin, and laughing gas were less commonly reported and cumulative dosages were much lower than self-reported lifetime use of ecstasy (number of tablets), cannabis (number of joints), amphetamine and cocaine (number of occasions), and alcohol and tobacco consumption (drinks/week vs cigarettes/week). Therefore, only ecstasy, amphetamine, cocaine, alcohol, and tobacco were used as independent variables.

Self-report histories may be inaccurate and in addition, there is imprecision arising from variation in drug content in ecstasy tablets. As a result, inaccuracies in dosage calculations are likely to undermine the validity of dose-response measures (Bedi and Redman, 2006). Also, drug use variables were far from normally distributed, even after log-transformation. Therefore, drug use variables were dichotomized using cutoff scores, which were fixed to balance the distribution of users and non-users of a particular drug within the sample. For ecstasy, amphetamines and cocaine the cutoff score was $>10$ tablets/ occasions lifetime. For cannabis, the cutoff score was set somewhat higher ( $>50$ joints lifetime), because experimenting with cannabis without becoming a frequent and long-term user is much more common than with the other illicit drugs. To illustrate this point, about half of the sample reported no use at all of ecstasy, amphetamines or cocaine, whereas only eight subjects reported no use at all of cannabis (see Table 2 for a description of the sample after drug use variables were dichotomized).

Performance data (dependent variables). Outcome measures included performance accuracy (for STERN, SAT, and PMT) and reaction times (STERN only). Task performance during STERN was measured by computing the decrease in reaction time (RT) owing to practice (mean RT during the novel task minus mean RT during the practiced task in $\mathrm{ms}$ ), and the decrease in error

Table I Demographic Features and Drug Use Characteristics

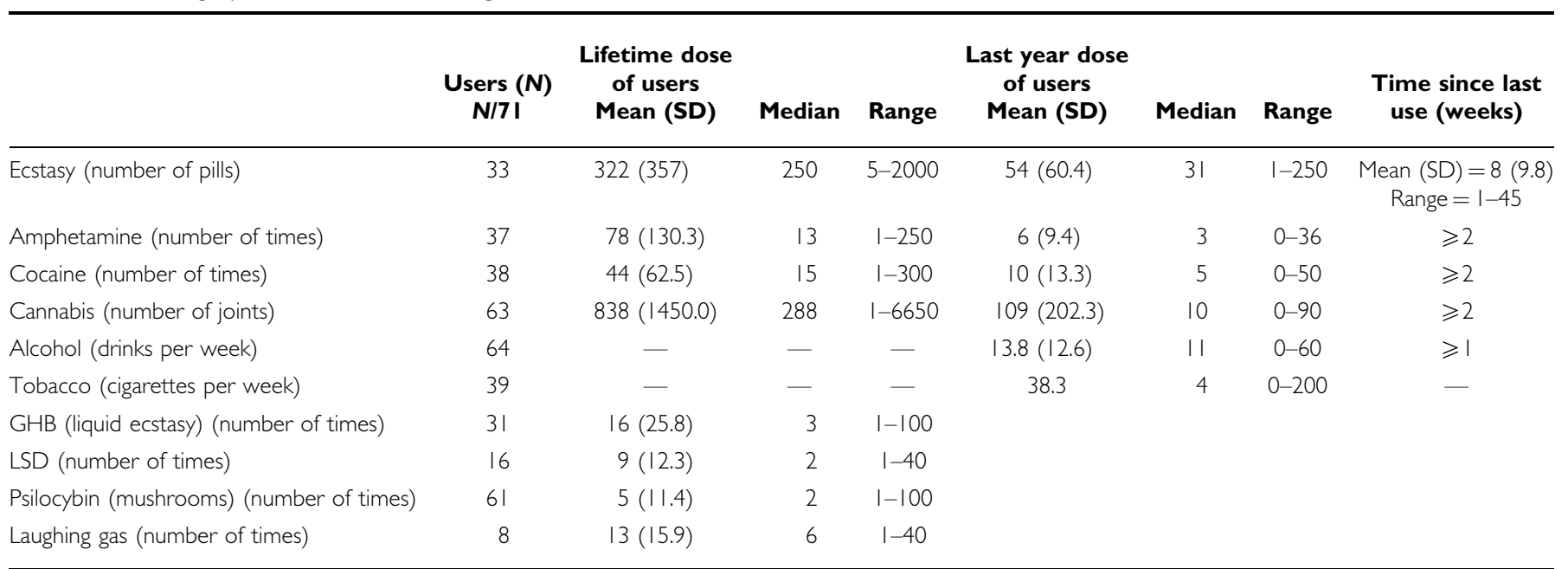

Mean (SD), median and range for the different drugs show scores from the users only 
rate owing to practice (errors novel task minus errors practiced task). Accuracy during SAT was calculated by dividing the number of correctly identified deviants by the total number of trials (percent correct). Recall accuracy during PMT was computed by averaging the percentage 'hits' (picture pairs correctly identified as seen previously) and 'correct negatives' (percentage of picture pairs correctly rejected as seen before) during the retrieval task.

fMRI (dependent variables). For all three tasks (STERN, SAT, and PMT) analysis of fMRI data involved the following stages: first, after motion correction, statistical activity maps were generated for each individual, for each of the task conditions compared with the rest condition by means of multiple regression (Worsley and Friston, 1995). Next, these maps were smoothed (FWHM $8 \mathrm{~mm}$ ) and normalized into standard MNI space (Collins et al, 1994), and were analyzed for the whole group, creating a contrast of interest for each task and using $z$-statistics (details are described in the given references for each task). We then identified regions of activity in the group-maps for each task, thresholded at $p<0.05$ Bonferroni corrected for the total number of voxels in the brain (Ramsey et al, 1996; Rypma et al, 2006). These group-maps were based on the following contrasts. For STERN, the regions of interest (ROIs) were derived from the difference between the novel and the CT. For SAT, the ROIs were obtained from the contrast between tone detection and rest. For PMT, the ROIs were based on the contrast between associative learning and simple classification.

For regression analyses (based on drug use), we used the mean levels of activity in each of these ROIs for each subject, for each task and for each condition within tasks, relative to resting state.

\section{Statistics}

Multiple regression analyses. Specific effects of ecstasy and the relative contributory effects of other substances (amphetamines, cocaine, cannabis, tobacco, and alcohol) on task performance and brain activity were assessed using multiple regression analyses with the different drugs as separate regressors. As non-drug predictors verbal IQ and gender were added to the model, because of the expected effect of IQ on cognitive task performance and because previous studies indicated a higher vulnerability to the effects of ecstasy in females (Reneman et al, 2001; Verheyden et al, 2002). Age was not included as a regressor owing to the relatively small age range within the sample, and to reduce the total number of regressors in the regression model (and thus maximizing statistical predictive power).

The strength of the effect of ecstasy use was estimated using two stepwise regression models. Model 1 estimated the crude or upper bound effect of ecstasy on neurocognitive function, that is, after adjustment for the effects of gender and IQ but without correction for the effects of other drugs. In the first step, gender and IQ were entered, then in a second step, ecstasy. The independent effect of ecstasy is quantified as the $R$-square change between the first and the second step of the model. This model resembles the approach in previous studies where ecstasy-users were compared with non-users without accounting for polydrug use. The effect of ecstasy in this model is likely to be an overestimation owing to the lack of correction for the impact of other drugs. Model 2 estimated the adjusted or lower bound effects of ecstasy with all other substance use, gender and IQ entered into the model first. Ecstasy use was entered as the second step, and its effect was expressed as the $R$-square change between the first and the second step.

Table 2 Description Sample After the Drug Use Variables were Dichotomised

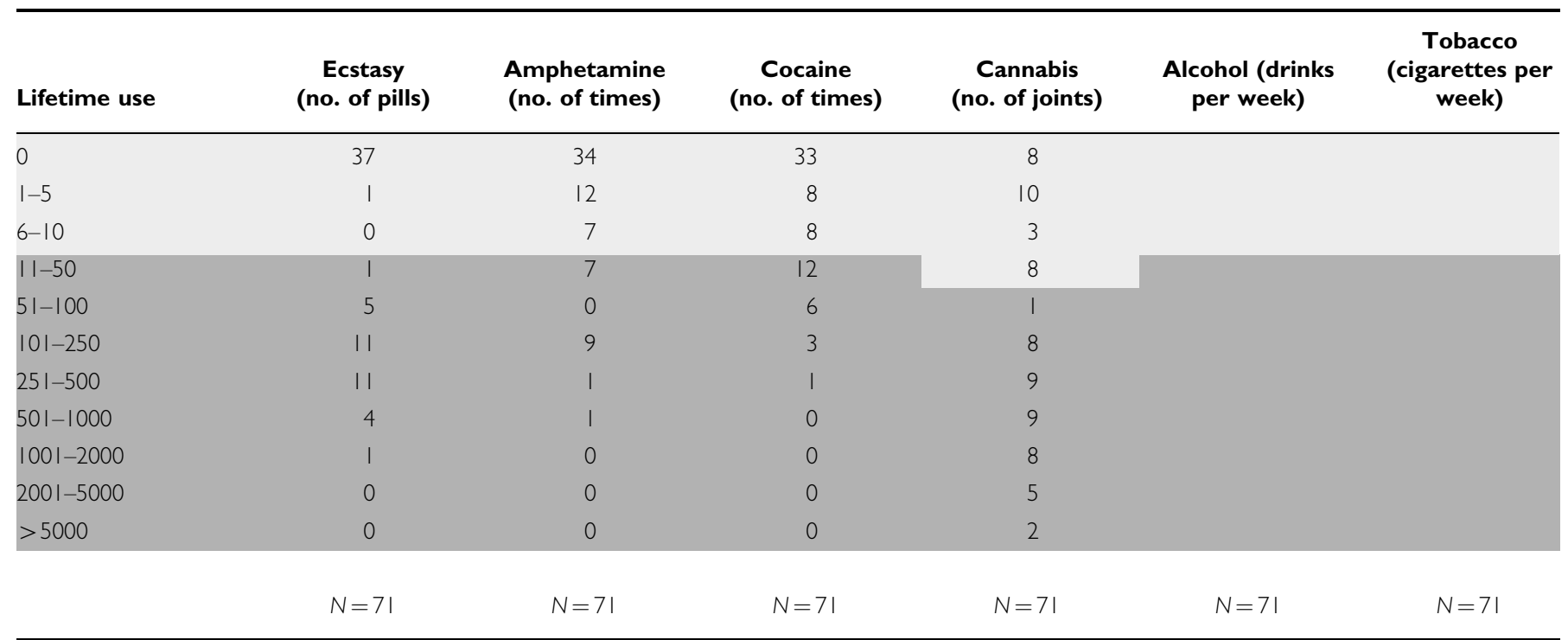

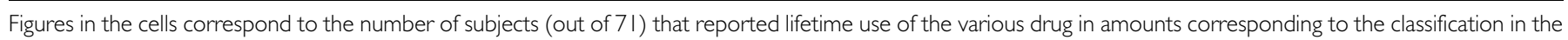

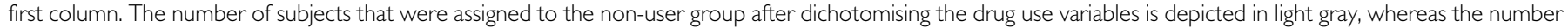
of subjects that were assigned to the user-group is depicted in dark gray.

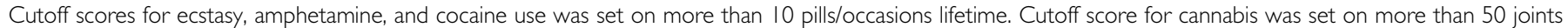
lifetime. Cutoff scores for alcohol and tobacco were set on more than 10 drinks and cigarettes per week, respectively. 
The effect of ecstasy in the second model is likely to be an underestimation.

Goodness-of-fit statistics were used to quantify the fit of the model and standardized regression coefficients- $\beta$ were used to indicate the predictive power of the different regressors.

Phi correlations were used to explore associations between dichotomized substance variables and demographic variables (see Table 3). The validity of the regression model is not affected by the relatively low associations (in terms of phi values) between some independent variables because each regressor in the stepwise model is adjusted for the predicting effect of all other regressors in the model. The variance inflation factor (VIF), a measure of multicollinearity, had a maximum value of 1.1 (as a rule of thumb any VIF that exceeds 10 is a reason for concern; Stevens, 1996), also indicating that correlations between variables in the model did not cause overspecification of the regression model.

\section{RESULTS}

\section{Sample Characteristics}

As data from a few tasks were lost for some subjects owing to technical malfunction, results are reported for each task separately with the number of subjects included within brackets. On average, mean verbal IQ was 101 (SD 7.7, range 83-122). Drug use characteristics are shown in Table 1 and 2 , and the correlations between use of various drugs are shown in Table 3.

\section{Performance}

Table 5 shows the results from multiple regression analyses for all three tasks. For performance during STERN model 2 yielded a marginal significant $\beta_{\text {ecstasy }}(R$-square ecstasy $=0.05$, $p=0.08$ ). However, the regression model as such failed to reach significance and, therefore, it was statistically not justified to interpret any of the separate standardized $\beta$ coefficients. For SAT performance neither regression model 1 nor model 2 reached significance. For PMT performance regression model 1 failed to explain a significant part of the variance in recall accuracy. Regression model 2 explained a marginally significant part $(R$-square $=20.3 \%, p=0.08)$ of the total variance in recall accuracy in the PMT task. However, ecstasy use explained only $1 \%$ (not significant) of the variance, whereas amphetamine use significantly predicted a reduction in recall accuracy $\left(\beta_{\text {amphetamine }}=-0.39\right.$, $p=0.007)$.

\section{fMRI}

The ROIs obtained with the contrast described in the methods are shown in Figure 4, and details are given in Table 4. The results of regression analyses are shown in Table 5. The key results are as follows: there were no significant effects of ecstasy or other illicit drugs on brain activity in the STERN $(n=70)$ or the SAT $(n=69)$ tasks. However, there were significant effects of tobacco use, gender, and IQ on the dorsolateral prefrontal cortex in the STERN task: being a smoker was associated with higher levels of brain activity in this brain area during working memory processing, as was being female, whereas higher IQ scores were associated with lower brain activity. Use of drugs did affect associative learning-related activity during the PMT $(n=69)$ task. Ecstasy use predicted lower activity in the left dorsolateral prefrontal cortex, whereas in the right middle occipital gyrus it was the opposite, that is stronger brain activity. Amphetamine use predicted lower activity in the right middle occipital gyrus, higher activity in the right dorsolateral prefrontal cortex and marginally lower activity in the left parahippocampal gyrus. Cocaine use predicted higher brain activity in the left dorsolateral prefrontal cortex and marginally higher activity in the right middle occipital gyrus. Finally, use of tobacco predicted marginally lower activity in the right middle occipital gyrus. Strength and direction of the effects of drug use on associative learning-related brain activity are depicted in Figure 5.

\section{DISCUSSION}

The present study found working memory and attention to be intact in polysubstance ecstasy users. However, polydrug use was associated with worse performance on an

Table 3 Correlations between Use of Various Drugs and Demographic Factors in Selected Sample $(N=71)^{\mathrm{a}}$

\begin{tabular}{|c|c|c|c|c|c|c|c|c|c|}
\hline & Age & Gender & IQ & Alcohol & Tobacco & Ecstasy & Amph & Cocaine & Cannabis \\
\hline Age & & NS & NS & NS & NS & NS & NS & NS & NS \\
\hline Gender & & & NS & NS & NS & NS & NS & NS & -0.22 \\
\hline DART-IQ & & & & NS & NS & NS & NS & NS & NS \\
\hline Alcohol & & & & & NS & NS & NS & NS & NS \\
\hline Tobacco & & & & & & 0.40 & NS & 0.24 & NS \\
\hline Ecstasy & & & & & & & 0.43 & 0.54 & NS \\
\hline Amphetamine & & & & & & & & 0.45 & NS \\
\hline Cocaine & & & & & & & & & NS \\
\hline Cannabis & & & & & & & & & \\
\hline
\end{tabular}

The Table shows the correlations (Phi, Pearson $\chi^{2}, p<0.05$, two-tailed) between age, gender $(0=$ male, $I=$ female) and the dichotomised drug use variables $(0=$ non-user, $I=$ user $)$. See Materials and methods section for classification criteria used. 
associative memory task. Interestingly, when effects of drugs were teased apart, it was the use of amphetamine, and not the use of ecstasy, that largely accounted for reduced associative memory performance.

Ecstasy use was related to altered brain activity patterns during associative learning in the left dorsolateral prefrontal cortex and the right middle occipital gyrus. These effects
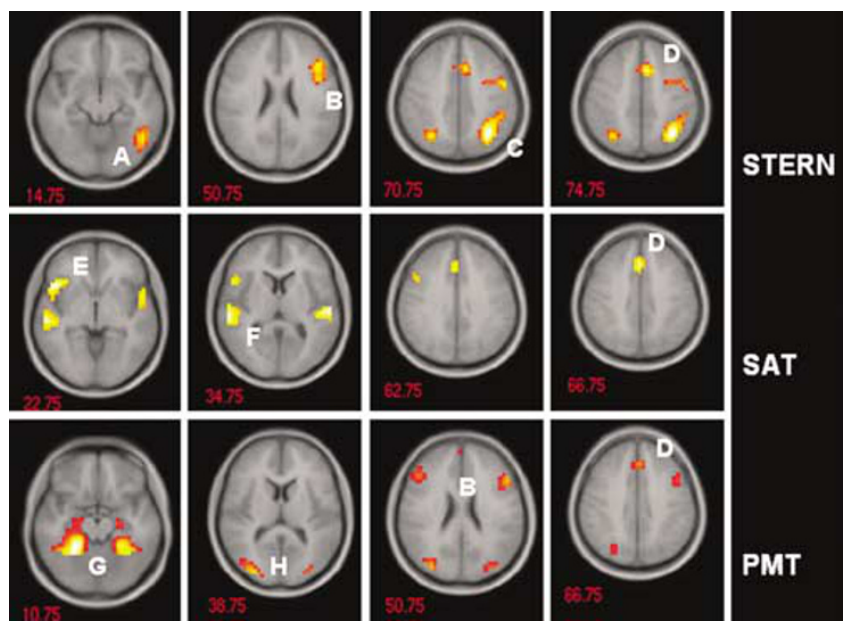

Figure 4 ROls for STERN, SAT and PMT: $A=$ fusiform gyrus, $\mathrm{B}=$ dorsolateral prefrontal cortex, $\mathrm{C}=$ superior parietal cortex, $\mathrm{D}=$ anterior cingulate cortex, $\mathrm{E}=$ inferior frontal cortex, $\mathrm{F}=$ auditory cortex, $\mathrm{G}=$ (para)hippocampal region, $\mathrm{H}=$ middle occipital gyrus. The numbers beneath the slices indicate the MNI $z$-coordinates. Slices are in radiological orientation (left side is right hemisphere and vice versa). were independent from those of cannabis and alcohol use, and appeared to be independent from those of amphetamine, cocaine, or tobacco use. These specific effects of ecstasy on brain activity during associative learning may reflect sustained, possibly long-term adaptation or compensatory reorganization in a fronto-visual network. Whether or not this signifies serotonin neurotoxicity in terms of neuronal damage cannot be concluded from the present findings. However, our results do not support the notion of widespread loss of serotonin axons, as the effects of ecstasy use were moderate, and selective for associative memory. It is therefore more likely that the network involved in associative memory is more sensitive to the effects of ecstasy on cognitive activation than other networks. Several underlying mechanisms may be involved. For one, ecstasy use could compromise serotonin function. Converging evidence from monkey and human studies suggests that the visual cortex is particularly sensitive to MDMA exposure. In a recent study by Brevard et al (2006), it was shown that a low dose of MDMA in marmoset monkeys had an intense effect on brain activity in the visual cortex without applied visual stimuli, measured with BOLD-fMRI. Similar BOLD-effects have been observed in human ecstasy users, that is, an increased visual cortex activation after photic stimulation where the magnitude of the increase was positively related to the degree of prior ecstasy exposure (Cowan et al, 2006). In addition, there is evidence of serotonergic modulation of functional brain activity in the prefrontal cortex and limbic structures during a cognitive challenge (Evers et al, 2005; Rubia et al, 2005). In one study, acute tryptophan depletion

Table 4 Regions of Interest

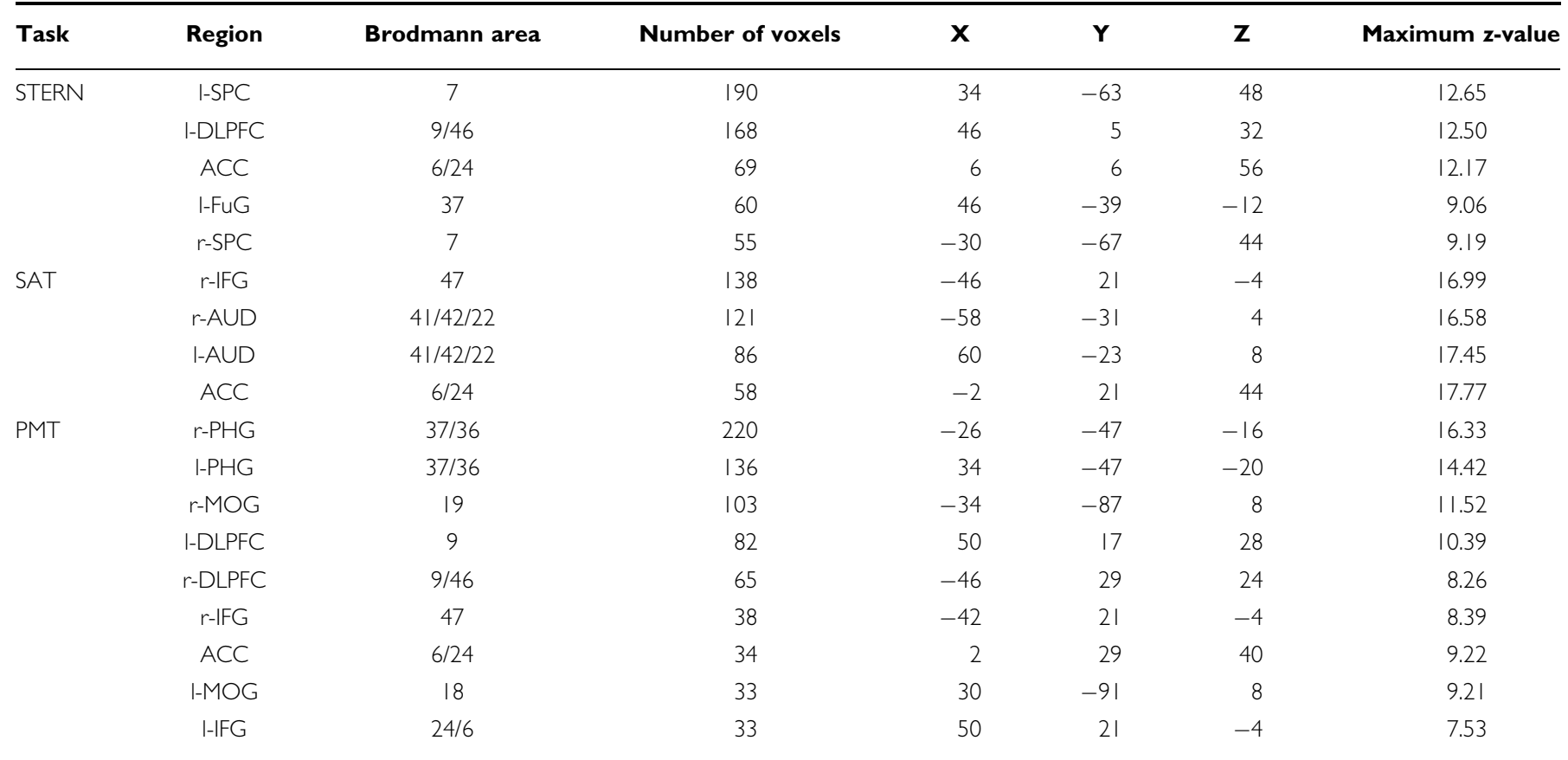

Abbreviations: 'I-' versus 'r-', 'left' versus 'right'; SPC, superior parietal cortex; DLPFC, dorsolateral prefrontal cortex; ACC, anterior cingulate cortex; FuG, fusiform gyrus; IFG, inferior frontal gyrus; AUD, auditory cortex; PHG, parahippocampal gyrus; MOG, middle occipital gyrus.

MNI coordinates are shown for the regions of interest for working memory (STERN), selective attention (SAT), and associative memory (PMT). The coordinates X, Y, and $Z$ represent location of the voxels with the highest $z$-value in the group map. Corresponding names and Brodmann areas are obtained from the location of voxels with the highest $z$-value. 
Table 5 Results Multiple Regression Analyses STERN, SAT and PMT

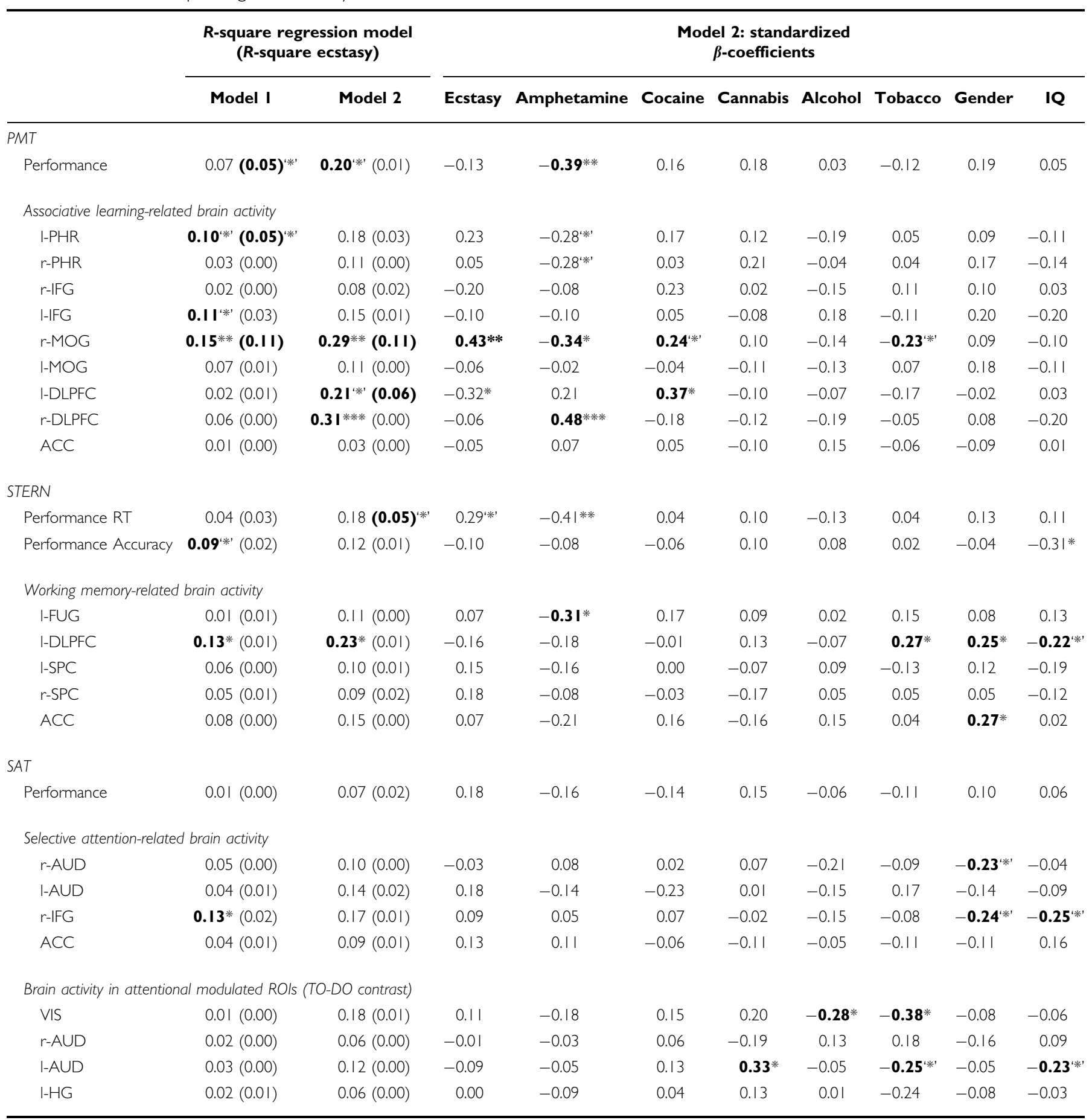

The Table shows the results from the regression analyses for PMT, STERN, and SAT, for Model I (upper bound effect ecstasy) and for Model 2 (lower bound effect ecstasy). For details on the regression models, see the paragraph on Statistics. The columns on Model I and Model 2 display the R-square of the overall regression model (reflecting the fit of the regression model). Significant or marginal significant $R$-square values are printed in bold. Within brackets the amount of variance uniquely explained by ecstasy use (R-square ecstasy) is shown, that is corrected for gender and verbal IQ (in Model I) or corrected for other substances, gender and verbal IQ (in Model 2). The right side of Table 5 shows the standardized $\beta$-coefficients of each predictor in Model 2. (Marginal) significant $\beta$-coefficients are printed in bold. Note: If Model 2 fails to reach significance, it is statistically not justified to interpret any of the separate $\beta$-coefficients and significant $\beta$-coefficients are NOT presented in bold. For abbreviations of the regions of interest see Table 4.

'*' $0.10<p<0.05 ; * p<0.05 ; * * * 0.01$; **** $p<0.001$.

significantly reduced brain activation in the right orbitoinferior prefrontal cortex, whereas it increased activation in the superior and medial temporal cortex. It was suggested that reduced prefrontal activation reflects low serotonin turnover, whereas the increased engagement of temporal brain regions could reflect compensatory mechanisms 
2. Amphetamine

3. Cocaine

4. Cannabis

5. Tobacco

⒍ Alcohol

$\square 7$. Gender

- 8. $\mathrm{IQ}$

$\begin{array}{ll}* * & =0.10<P<0.05 \\ * & =P<0.05 \\ * & =P<0.01 \\ * * * & =P<0.001\end{array}$

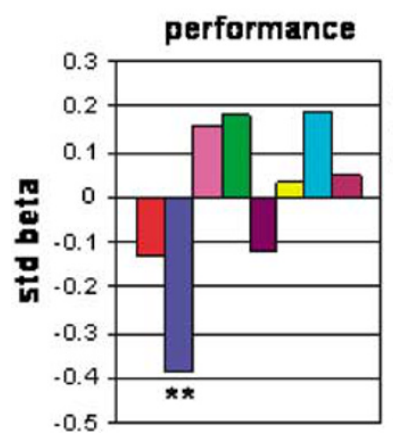

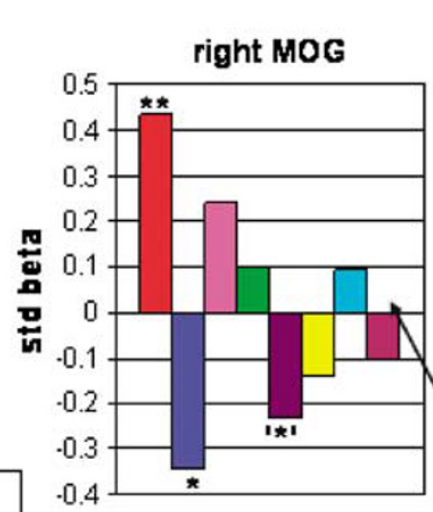

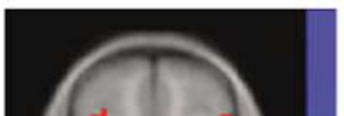

$-10.2$

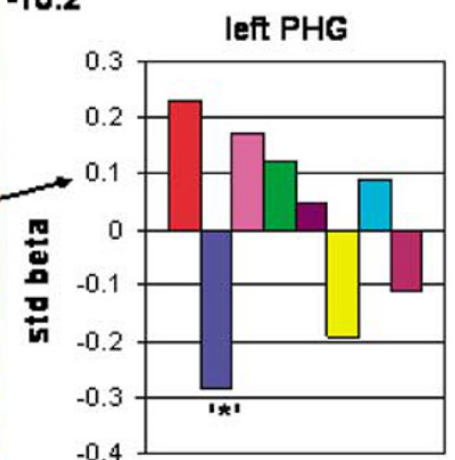

5.5

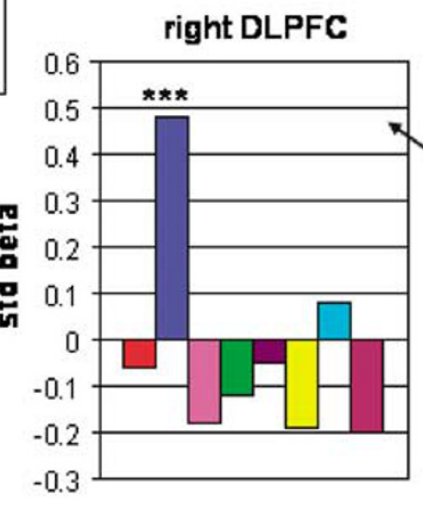

right DLPFC
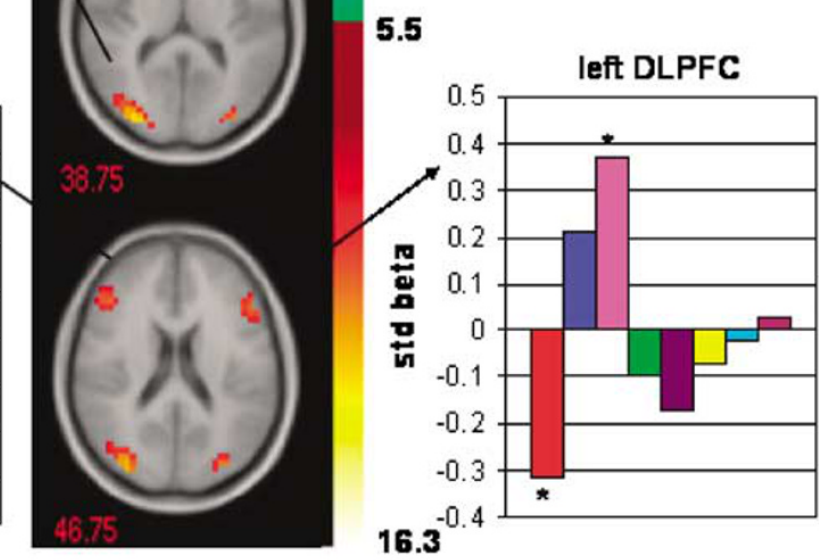

Figure 5 Overview of the results from the multiple regression analyses for PMT. The vertical axis of the graphs represents standardized $\beta$-coefficients: values reflect strength and direction of the association between use of the drug and magnitude of brain activity in a brain region. On the horizontal axis, colored bars represent the different drugs (see legend; number I-8 displayed from left to right in the graphs). Abbreviations: L-PHR = left parahippocampal region, L-DLPFC = left dorsolateral prefrontal cortex, R-DLPFC = right dorsolateral prefrontal cortex, R-MOG = right middle occipital gyrus. The numbers beneath the slices indicate the $\mathrm{MNI} z$-coordinates. Slices are in radiological orientation (left side is right hemisphere and vice versa).

(Rubia et al, 2005). These findings bear a resemblance to the current observations of and reduced activation of the dorsolateral prefrontal cortex, increased activation of the middle occipital gyrus during associative learning. Thus, it is conceivable that heavy ecstasy use induces sustained reduced serotonin turnover in the prefrontal cortex. However, this does not explain why lower prefrontal activation in ecstasy users was selective to one cognitive domain, that is associative memory; the left dorsolateral prefrontal cortex, activated in all tasks (see Figure 4, ROI 'B' for STERN and PMT, data not shown for SAT), was only affected during associative memory. Another mechanism could be mild damage to (part of) the associative memory system owing to ecstasy use, though serious enough to lead to reduced functioning. In this case, the (para)hippocampal region is the most plausible candidate as this region was only activated during associative memory, and not during working memory or attention. However, our results suggest that the (para)hippocampal region may not be a prime target for ecstasy-related neurotoxicity (Daumann et al, 2005), as we found only tentative evidence for enhanced brain activity in the left parahippocampal area.

In addition to the specific effects of ecstasy, the effects of amphetamine on activity in the network engaged during associative memory are of special interest as most of the heavy ecstasy users also take amphetamine. Amphetamine use was related to altered brain activity in the right middle occipital cortex, the right dorsolateral prefrontal cortex and possibly the left parahippocampal region (statistical trend). It should be noted that the effects of ecstasy and amphetamine on brain activation were in the opposite direction in the prefrontal and middle occipital regions, suggesting that different mechanisms are at play. This could be related to the different neurotransmitter systems these drugs predominantly act upon, namely, the serotonin (ecstasy) and the dopamine (amphetamine) system. At present, there is no compelling evidence to suggest that ecstasy use damages dopamine neurons in the human brain (Colado et al, 2004), but there is evidence from neuroimaging studies that (meth)amphetamine can induce changes in dopaminergic brain circuits (Choi et al, 2006; Reneman et al, 2002; Uftring et al, 2001; Volkow et al, 2001). However, more research with other (neuroimaging) techniques is necessary to further elucidate the neurochemical mechanisms underlying the drug-induced alterations in the BOLD signal, as measured in the present study.

Our findings of impaired associative memory performance in polysubstance (ecstasy) users are consistent with 
many neuropsychological studies reporting deficits in mainly verbal memory tests in heavy ecstasy users. It is important to note, however, that our results showed that lower associative memory performance in ecstasy users was largely due to amphetamine, and not ecstasy use. As previous studies in heavy ecstasy users often contend with confounding effects of polysubstance use (including amphetamine), an alternative explanation could be that (part of) the previously observed impairments in memory may be related to concomitant use of amphetamine. Furthermore, our brain activity data challenge the notion that memory impairments in polysubstance ecstasy users reflect hippocampal dysfunction owing to a specific vulnerability of this brain region to the neurotoxic effects of ecstasy (Daumann et al, 2005; Jacobsen et al, 2004). Only amphetamine seemed to affect the parahippocampal region to some extent, but there was no effect of ecstasy.

With regard to working memory and attention our results are less straightforward. We found no evidence for robust effects of ecstasy or other illicit drugs on performance or brain activity in the networks engaged during working memory or selective attention. This seems at odds with findings from several previous fMRI studies on working memory in ecstasy users in which both decreased and enhanced brain activity has been reported in ecstasy users in a variety of brain regions, including the frontal and temporal cortex (Daumann et al, 2003, 2004a, b, c, d; Jacobsen et al, 2004; Moeller et al, 2004). There are several possible explanations for the discrepancy between the current and previous findings: first, activity in brain areas other than those consistently reported as being activated in working memory studies in healthy controls, that is parietal and frontal areas (D'Esposito et al, 1998), may result from specific characteristics of the fMRI task paradigm used. Consequently, observed differences between ecstasy users and non-users in, for example, hippocampal activity, may not be specifically related to working memory, but may surface because the task paradigm used involves other (episodic) memory processes as well. Second, differences in statistical analyses impede comparisons between the studies. In the present study, we used a whole brain analysis correcting for multiple comparisons, whereas other studies used more liberal statistical thresholds (Daumann et al, 2003) or performed a ROI analysis restricted to the (para)hippocampal region (Jacobsen et al, 2004). This may have biased results to certain brain areas that are not involved in working memory per se.

Several limitations of the current study should be noted. For one, a consistent critique of a cross-sectional design is that neurocognitive abnormalities might actually predate and place individuals at risk for drug abuse rather than being the result of abuse. In this regard, it is important to note that animal research has reported MDMA-induced neurotoxicity in several species, including primates (Ricaurte et al, 2000). Furthermore, several human studies have demonstrated dose-effect relationships between cumulative lifetime ecstasy use and memory deficits, which support the idea of ecstasy use causing neurocognitive impairments (Fox et al, 2001). A second limitation is that we had to rely on statements by the subjects themselves on their current and earlier consumption habits, with questionable reliability. Unfortunately, in a naturalistic design there is no obvious solution to this problem. Drug hair analyses confirmed previous ecstasy use in $86 \%$ of the subjects who reported to have used ecstasy. In addition, the results from hair analyses showed no evidence for previous use of ecstasy in $96 \%$ of the subjects who had reported to be ecstasy-naive. Drug hair analysis yields no information on patterns of ecstasy use, that is frequency, dosage (eg multiple pill ingestion per occasion resulting in nonlinear MDMA plasma levels), or cumulative dose lifetime, but the results support the plausibility of self-reported data on ecstasy use in the current study. Pill purity is related to reliability of self-reported drug use data. Pills containing pure MDMA are probably rare. Nevertheless, between 2002 and 2004, when subjects were recruited, pill-testing confirms that in The Netherlands more than $95 \%$ of the tablets sold as ecstasy contain MDMA as the sole (91.2\%) or main (4.2\%) psychoactive component (Netherlands National Drug Monitor, 2004 (http://www.trimbos.nl/)). A third limitation is that despite the substantial time and effort spent on recruitment, sample stratification was not completely satisfactory. The resulting correlations between use of ecstasy and amphetamine and cocaine were reduced but still substantial and statistically significant. Thus, we cannot claim full independence between predictors. Nonetheless, correlations between use of ecstasy and use of other illicit drugs were lower than usually found after random recruitment among frequent ecstasy users (Scholey et al, 2004; Parrott et al, 2002) and multicollinearity diagnostics indicated that the regression model allowed for reliable estimation of the effects of the various drugs. In contrast, the association between ecstasy use and its most commonly used co-drug cannabis was successfully removed as a result of sample stratification, thereby controlling for an important potential confounder.

In conclusion, this study does not show strong effects of use of ecstasy or other drugs in the domains of working memory and attention. However, polysubstance ecstasy users exhibit reduced associative memory performance, but this impairment is largely due to concomitant amphetamine use, and not to ecstasy use. Moreover, ecstasy and amphetamine have differential and partly opposite effects on brain activity in the network engaged during associative memory, suggesting that different mechanisms are at play that could be related to differential neurochemical effects on serotonin and dopamine systems. The effects of ecstasy and amphetamine are not specific for brain structures; the dorsolateral prefrontal cortex, activated in all tasks, is only affected during associative memory. It seems more likely that the network as a whole is affected, and that the dorsolateral prefrontal cortex responds differently to alterations in serotonergic $v s$ dopaminergic neurotransmission within certain brain systems.

\section{ACKNOWLEDGEMENTS}

This research was supported by a grant of The Netherlands Organisation for Health Research and Development as part of their Addiction Program (ZonMW 310-00-036). We thank Margriet Sitskoorn and Marieke Langen for their contribution to the PMT task. We thank Hylke Vervaeke, Jeske Damoiseaux, Mieke Deenen, Judith Bosman, and 
Rianne Petersen for their assistance with subject recruitment and data collection, and Erika van Hell and Lenny Ramsey for their assistance with data preprocessing. Finally, we thank Maarten Koeter, Ben Schmand, Dirk Korf, and Jan Booij for their valuable comments on statistical analysis and this paper.

\section{REFERENCES}

Bedi G, Redman J (2006). Self-reported ecstasy use: the impact of assessment method on dosage estimates in recreational users. J Psychopharmacol 20: 432-436.

Brevard ME, Meyer JS, Harder JA, Ferris CF (2006). Imaging brain activity in conscious monkeys following oral MDMA ('ecstasy'). Magn Reson Imaging 24: 707-714.

Buchert R, Thomasius R, Wilke F, Petersen K, Nebeling B, Obrocki $\mathrm{J}$ et al (2004). A voxel-based PET investigation of the long-term effects of 'Ecstasy' consumption on brain serotonin transporters. Am J Psychiatry 161: 1181-1189.

Choi JK, Chen YI, Hamel E, Jenkins BG (2006). Brain hemodynamic changes mediated by dopamine receptors: role of the cerebral microvasculature in dopamine-mediated neurovascular coupling. Neuroimage 30: 700-712.

Colado MI, O'Shea E, Green AR (2004). Acute and long-term effects of MDMA on cerebral dopamine biochemistry and function. Psychopharmacology (Berlin) 173: 249-263.

Collins DL, Neelin P, Peters TM, Evans AC (1994). Automatic 3D intersubject registration of MR volumetric data in standardized Talairach space. J Comput Assist Tomogr 18: 192-205.

Cowan RL, Haga E, Frederick BB, Dietrich MS, Vimal RL, Lukas SE et al (2006). MDMA use is associated with increased spatial BOLD fMRI visual cortex activation in human MDMA users. Pharm Biochem Behav 84: 219-228.

Daumann J, Fischermann T, Heekeren K, Henke K, Thron A, Gouzoulis-Mayfrank E (2005). Memory-related hippocampal dysfunction in poly-drug ecstasy (3,4-methylenedioxymethamphetamine) users. Psychopharmacology (Berlin) 180: 607-611.

Daumann J, Fischermann T, Heekeren K, Thron A, GouzoulisMayfrank E (2004a). Neural mechanisms of working memory in ecstasy (MDMA) users who continue or discontinue ecstasy and amphetamine use: evidence from an 18-month longitudinal functional magnetic resonance imaging study. Biol Psychiatry 56: 349-355.

Daumann J, Fischermann T, Heekeren K, Thron A, GouzoulisMayfrank E (2004b). Neural mechanisms of working memory in ecstasy (MDMA) users who continue or discontinue ecstasy and amphetamine use: evidence from an 18-month longitudinal functional magnetic resonance imaging study. Biol Psychiatry 56: 349-355.

Daumann J, Hensen G, Thimm B, Rezk M, Till B, GouzoulisMayfrank E (2004c). Self-reported psychopathological symptoms in recreational ecstasy (MDMA) users are mainly associated with regular cannabis use: further evidence from a combined crosssectional/longitudinal investigation. Psychopharmacology (Berlin) 173: 398-404.

Daumann J, Hensen G, Thimm B, Rezk M, Till B, GouzoulisMayfrank E (2004d). Self-reported psychopathological symptoms in recreational ecstasy (MDMA) users are mainly associated with regular cannabis use: further evidence from a combined cross-sectional/longitudinal investigation. Psychopharmacology 173: 398-404.

Daumann J, Schnitker R, Weidemann J, Schnell K, Thron A, Gouzoulis-Mayfrank E (2003). Neural correlates of working memory in pure and polyvalent ecstasy (MDMA) users. Neuroreport 14: 1983-1987.

De Win MM, Jager G, Vervaeke HK, Schilt T, Reneman L, Booij J et al (2005). The Netherlands XTC Toxicity (NeXT) study: objectives and methods of a study investigating causality, course, and clinical relevance. Int J Methods Psychiatr Res 14: 167-185. De Win MM, Reneman L, Jager G, Vlieger EP, Olabarriaga SD, Lavini C et al (2007). A prospective cohort study on sustained effects of low-dose ecstasy use on the brain in new ecstasy users. Neuropsychopharmacology 32: 458-470.

D'Esposito M, Aguirre GK, Zarahn E, Ballard D, Shin RK, Lease J (1998). Functional MRI studies of spatial and nonspatial working memory. Brain Res Cogn Brain Res 7: 1-13.

Evers EA, Cools R, Clark L, van der Veen FM, Jolles J, Sahakian BJ et al (2005). Serotonergic modulation of prefrontal cortex during negative feedback in probabilistic reversal learning. Neuropsychopharmacology 30: 1138-1147.

Fox HC, Parrott AC, Turner JJ (2001). Ecstasy use: cognitive deficits related to dosage rather than self-reported problematic use of the drug. J Psychopharmacol 15: 273-281.

Gouzoulis-Mayfrank E, Daumann J (2006a). The confounding problem of polydrug use in recreational ecstasy/MDMA users: a brief overview. J Psychopharmacol 20: 188-193.

Gouzoulis-Mayfrank E, Daumann J (2006b). Neurotoxicity of methylenedioxyamphetamines (MDMA; ecstasy) in humans: how strong is the evidence for persistent brain damage? Addiction 101: 348-361.

Green AR, Mechan AO, Elliott JM, O'Shea E, Colado MI (2003). The pharmacology and clinical pharmacology of 3,4-methylenedioxymethamphetamine (MDMA, 'ecstasy'). Pharmacol Rev 55: 463-508.

Halpern JH, Pope Jr HG, Sherwood AR, Barry S, Hudson JI, Yurgelun-Todd D (2004). Residual neuropsychological effects of illicit 3,4-methylenedioxymethamphetamine (MDMA) in individuals with minimal exposure to other drugs. Drug Alcohol Depend 75: 135-147.

Henke K, Buck A, Weber B, Wiesser HG (1997). Human hippocampus establishes associations in memory. Hippocampus 7: 249-256.

Jacobsen LK, Mencl WE, Pugh KR, Skudlarski P, Krystal JH (2004). Preliminary evidence of hippocampal dysfunction in adolescent MDMA ('ecstasy') users: possible relationship to neurotoxic effects. Psychopharmacology (Berlin) 173: 383-390.

Jager G, Kahn RS, van den Brink W, van Ree JM, Ramsey NF (2006). Long-term effects of frequent cannabis use on working memory and attention: an fMRI study. Psychopharmacology (Berlin) 185: 358-368.

Jager G, van Hell HH, de Win MM, Kahn RS, van den Brink W, van Ree JM et al (2007). Effects of frequent cannabis use on hippocampal activity during an associative memory task. Eur Neuropsychopharmacol 17: 289-297.

Jansma JM, Ramsey NF, Slagter HA, Kahn RS (2001). Functional anatomical correlates of controlled and automatic processing. J Cogn Neurosci 13: 730-743.

Kish SJ (2002). How strong is the evidence that brain serotonin neurons are damaged in human users of ecstasy? Pharmacol Biochem Behav 71: 845-855.

McCann UD, Szabo Z, Scheffel U, Dannals RF, Ricaurte GA (1998). Positron emission tomographic evidence of toxic effect of MDMA ('Ecstasy') on brain serotonin neurons in human beings. Lancet 352: 1433-1437.

McCann UD, Szabo Z, Seckin E, Rosenblatt P, Mathews HB, Ravert $\mathrm{HT}$ et al (2005). Quantitative PET studies of the serotonin transporter in MDMA users and controls using [11C]McN5652 and [11C]DASB. Neuropsychopharmacology 30: 1741-1750.

Medina KL, Shear PK, Corcoran K (2005). Ecstasy (MDMA) exposure and neuropsychological functioning: a polydrug perspective. J Int Neuropsychol Soc 11: 753-765.

Moeller FG, Steinberg JL, Dougherty DM, Narayana PA, Kramer LA, Renshaw PF (2004). Functional MRI study of working memory in MDMA users. Psychopharmacology (Berlin) 177: 185-194. 
Morgan MJ (2000). Ecstasy (MDMA): a review of its possible persistent psychological effects. Psychopharmacology 152: 230-248.

Morton J (2005). Pharmacology and neurotoxicity. Curr Opin Pharmacol 5: 79-86.

Parrott AC (2000). Human research on MDMA (3,4-methylenedioxymethamphetamine) neurotoxicity: cognitive and behavioural indices of change. Neuropsychobiology 42: 17-24.

Parrott AC (2001). Human psychopharmacology of Ecstasy (MDMA): a review of 15 years of empirical research. Hum Psychopharmacol 16: 557-577.

Parrot AC (2006). MDMA in humans: factors which affect the neuropsychobiological profiles of recreational ecstasy users, the integrative role of bioenergetic stress. J Psychopharmacol 20: 417-424.

Parrott AC, Buchanan T, Scholey AB, Heffernan T, Ling J, Rodgers J (2002). Ecstasy/MDMA attributed problems reported by novice, moderate and heavy recreational users. Hum Psychopharmacol 17: 309-312.

Ramsey NF, Jansma JM, Jager G, Van Raalten T, Kahn RS (2004). Neurophysiological factors in human information processing capacity. Brain 127: 517-525.

Ramsey NF, Kirkby BS, van Gelderen P, Berman KF, Duyn JH, Frank JA et al (1996). Functional mapping of human sensorimotor cortex with 3D BOLD fMRI correlates highly with H2(15)O PET rCBF. J Cereb Blood Flow Metab 16: 755-764.

Ramsey NF, van den Brink JS, van Muiswinkel AM, Folkers PJ, Moonen CT, Jansma JM et al (1998). Phase navigator correction in 3D fMRI improves detection of brain activation: quantitative assessment with a graded motor activation procedure. Neuroimage 8: $240-248$.

Reneman L (2003). Designer drugs: how dangerous are they? J Neural Transm Suppl 66: 61-83.

Reneman L, Booij J, de Bruin K, Reitsma JB, de Wolff FA, Gunning WB et al (2001). Effects of dose, sex, and long-term abstention from use on toxic effects of MDMA (ecstasy) on brain serotonin neurons. Lancet 358: 1864-1869.

Reneman L, Booij J, Lavalaye J, de Bruin K, Reitsma JB, Gunning B et al (2002). Use of amphetamine by recreational users of ecstasy (MDMA) is associated with reduced striatal dopamine transporter densities: a [123I] beta-CIT SPECT study-preliminary report. Psychopharmacology (Berl) 159: 335-340.

Reneman L, de Win MM, van den Brink W, Booij J, den Heeten GJ (2006). Neuroimaging findings with MDMA/ecstasy: technical aspects, conceptual issues and future prospects. J Psychopharmacol 20: 164-175.

Ricaurte GA, Yuan J, McCann UD (2000). (+/-)3,4-Methylenedioxymethamphetamine ('Ecstasy')-induced serotonin neurotoxicity: studies in animals. Neuropsychobiology 42: 5-10.

Rodgers J (2000). Cognitive performance amongst recreational users of 'ecstasy'. Psychopharmacology (Berlin) 151: 19-24.

Rubia K, Lee F, Cleare AJ, Tunstall N, Fu CH, Brammer M et al (2005). Tryptophan depletion reduces right inferior prefrontal activation during response inhibition in fast, event-related fMRI. Psychopharmacology (Berlin) 179: 791-803.

Rypma B, Berger JS, Prabhakaran V, Martin Bly B, Kimberg DY, Biswall BB et al (2006). Neural correlates of cognitive efficiency. Neuroimage 33: 969-979.

Schilt T, de Win MM, Koeter M, Jager G, Korf DJ, van den Brink W et al. Cognition in novice Ecstasy users with minimal exposure to other drugs: a prospective cohort study. Arch Gen Psychiatry (in press).

Schmand B, Bakker D, Saan R, Louman J (1991). The dutch reading test for adults: a measure of premorbid intelligence level. Tijdschr Gerontol Geriatr 22: 15-19.

Scholey AB, Parrott AC, Buchanan T, Heffernan TM, Ling J, Rodgers J (2004). Increased intensity of Ecstasy and polydrug usage in the more experienced recreational Ecstasy/MDMA users: a WWW study. Addict Behav 29: 743-752.

Semple DM, Ebmeier KP, Glabus MF, O'Carroll RE, Johnstone EC (1999). Reduced in vivo binding to the serotonin transporter in the cerebral cortex of MDMA ('ecstasy') users. Br J Psychiatry 176: 193-195.

Sheehan DV, Lecrubier Y, Sheehan KH, Amorim P, Janavs J, Weiller E et al (1998). The Mini-International Neuropsychiatric Interview (M.I.N.I.): the development and validation of a structured diagnostic psychiatric interview for DSM-IV and ICD-10. J Clin Psychiatry 59(Suppl 20): 22-33.

Stevens J (1996). Applied Multivariate Statistics for the Social Sciences, 3rd edn. Lawrence Erlbaum Associates: Mahwah, New Jersey. pp 64-83.

Turner JJ, Parrott AC (2000). Is MDMA a human neurotoxin? diverse views from the discussants. Neuropsychobiology 42: 42-48.

Uftring SJ, Wachtel SR, Chu D, McCandless C, Levin DN, de Wit H (2001). An fMRI study of the effect of amphetamine on brain activity. Neuropsychopharmacology 25: 925-935.

Van den Wijngaart G, Braam R, de Bruin D, Fris N, Maalste M et al (1997). Ecstasy and the Dutch rave scene: A socio-epidemiologic study on the nature and extent of, and the risks involved in using ecstasy and other party drugs at dance events. Addiction Research Center Utrecht: The Netherlands.

Verbaten MN (2003). Specific memory deficits in ecstasy users? The results of a meta-analysis. Hum Psychopharmacol 18: 281-290.

Verheyden SL, Hadfield J, Calin T, Curran HV (2002). Sub-acute effects of MDMA (+/-3,4-methylenedioxymethamphetamine, 'ecstasy') on mood: evidence of gender differences. Psychopharmacology (Berlin) 161: 23-31.

Volkow ND, Chang L, Wang GJ, Fowler JS, Franceschi D, Sedler MJ et al (2001). Higher cortical and lower subcortical metabolism in detoxified methamphetamine abusers. Am J Psychiatry 158: 383-389.

Worsley KJ, Friston KJ (1995). Analysis of fMRI time-series revisited-again. Neuroimage 2: 173-181. 